PROCEEDINGS OF THE

AMERICAN MATHEMATICAL SOCIETY

Volume 132, Number 1, Pages 231-237

S 0002-9939(03)06997-1

Article electronically published on May 9, 2003

\title{
SOME REMARKS ON TOTALLY IMPERFECT SETS
}

\author{
ANDRZEJ NOWIK AND TOMASZ WEISS
}

(Communicated by Carl G. Jockusch, Jr.)

Abstract. We prove the following two theorems.

Theorem 1. Let $X$ be a strongly meager subset of $2^{\omega \times \omega}$. Then it is dual Ramsey null.

We will say that a $\sigma$-ideal $\mathcal{I}$ of subsets of $2^{\omega}$ satisfies the condition ( $\ddagger$ ) iff for every $X \subseteq 2^{\omega}$, if

$$
\forall_{f \in \omega \uparrow \omega}\left\{g \in \omega^{\uparrow \omega}: \neg(f \prec g)\right\} \cap X \in \mathcal{I},
$$

then $X \in \mathcal{I}$

Theorem 2. The $\sigma$-ideals of perfectly meager sets, universally meager sets and perfectly meager sets in the transitive sense satisfy the condition ( $)$.

\section{Preliminaries}

In this paper we continue our investigation of totally imperfect subsets of $2^{\omega}$ (see [N], [NW1], and [NW2] for related results).

We divide it into two parts, the first one is devoted to some properties of strongly meager sets and the second one deals with combinatorial properties of perfectly meager sets.

We use standard set theoretic notation. We recall here the main notation and definitions from the paper $\mathrm{CS}$.

By a partition we mean a collection of pairwise disjoint, nonempty subsets of $\omega$ whose union is $\omega$. By $(\omega)^{\leq \omega},(\omega)^{<\omega}$ and $(\omega)^{\omega}$ we denote the set of all partitions, the set of all finite partitions and the set of all infinite partitions, respectively. If $X, Y \in(\omega)^{\leq \omega}$, then we say that $Y$ is coarser than $X$ iff $\forall_{A \in X} \exists_{B \in Y} A \subseteq B$ and we write $Y \leq X$.

For any collection of sets $\mathcal{A}$, let us define $\|\mathcal{A}\|=\bigcup \mathcal{A}$. For $X \in(\omega)^{\omega}$ and $n \in \omega$, we put $X[n]=\{x \cap n: x \in X\} \backslash\{\emptyset\}$. Suppose that $s$ is a (finite) partition of some $n \in \omega$. We write $s \prec X$ iff $s=X[n]$, where $n=\|s\|$. We say that $s \leq t$ iff $\|s\|=\|t\|$ and $s$ is coarser than $t$. Finally, $s \leq X$ iff $s \leq X[\|s\|]$. For every $s, X$ such that $s \leq X$, we define $(s, X)=\left\{Y \in(\omega)^{\omega}: s \prec Y \leq X\right\}$, and following [CS] we call it a dual Ellentuck neighborhood.

A set $D \subseteq(\omega)^{\omega}$ is said to be a dual Ramsey null iff for each dual Ellentuck neighborhood $(s, X)$, there exists $Y \in(s, X)$ such that $(s, Y) \cap D=\emptyset$.

Received by the editors March 14, 2002 and, in revised form, August 19, 2002.

2000 Mathematics Subject Classification. Primary 03E15; Secondary 03E20, 28E15.

Key words and phrases. Strongly meager sets, dual Ramsey null sets, partitions. 
Notice that each $X \in(\omega)^{\omega}$ can be identified with an element $\tilde{X} \in 2^{\omega \times \omega}$ by the following correspondence: $\tilde{X}(\langle m, n\rangle)=1$ iff $\exists_{x \in X} m, n \in x$. Thus, we can assume that $(\omega)^{\omega}$ is a subset of $2^{\omega \times \omega}$.

We recall that $E \subseteq 2^{\omega \times \omega}$ is said to be strongly meager iff for each measure zero set $N$ included in $2^{\omega \times \omega}, E+N \neq 2^{\omega \times \omega}$ (see for example [BJ]).

\section{Strongly meager Sets}

The proof of our main result of this part is based on the following well-known Lorentz's theorem (see $[\mathrm{L}]$ ) which has many interesting applications in the area of special subsets of the reals (see, for example, [NSW] and [NW2]).

Theorem 1.1 (Lorentz). If $(G,+)$ is a finite group and if $H$ is a nonempty subset of $G$, then there is a subset $F$ of $G$ such that

(1) the cardinality of $F$ is at most $|G| \cdot\left(\frac{1+\ln (|H|)}{|H|}\right)$, and

(2) $G=F+H$.

Lemma 1.2. For any dual Ellentuck neighborhood $(s, A)$, there exists a measure zero set $H \in \mathcal{N}\left(2^{\omega \times \omega}\right)$ such that for each $t \in 2^{\omega \times \omega}$, one can find $B \in(s, A)$ such that $(s, B) \subseteq H+t$.

Proof. For every $n \in \omega$, choose $K_{n} \in \omega$ such that

$$
\frac{\ln \left(K_{n}\right)+1}{K_{n}} \leq \frac{1}{2^{n}}
$$

Since one can easily find a partition $A^{\prime} \in(s, A)$ such that $\forall_{x \in A^{\prime}}|x|=\omega$, we may further assume, without loss of generality, that $\forall_{x \in A}|x|=\omega$. Let $\left(A_{n}^{(k)}\right)_{n \in \omega, 0 \leq k \leq K_{n}}$ be an enumeration of all elements of the set $A \backslash\{x \in A: x \cap\|s\| \neq \emptyset\}$. For each $n, k \in \omega$ pick an arbitrary $a_{n}^{(k)} \in A_{n}^{(k)}$. Next, for every $n \in \omega$, define

$$
I_{n}=\left\{a_{n}^{(k)}: 0 \leq k \leq K_{n}\right\}
$$

and $J_{n}=I_{n} \times I_{n}$. For each $n \in \omega$ and $k \in\left[0, K_{n}\right)$, let a function $b_{n}^{(k)}: J_{n} \rightarrow 2$ be defined by the following conditions:

$$
b_{n}^{(k)}\left(\left\langle m_{1}, m_{2}\right\rangle\right)= \begin{cases}1 & \text { if } k\left(m_{1}\right), k\left(m_{2}\right) \leq k \vee k\left(m_{1}\right), k\left(m_{2}\right)>k, \\ 0 & \text { otherwise, }\end{cases}
$$

where $k(m)$ denotes a (unique) $k \in\left[0, K_{n}\right]$ such that $m \in A_{n}^{(k)}$. Put

$$
B_{n}=\left\{b_{n}^{(k)}: 0 \leq k<K_{n}\right\} .
$$

Obviously, $B_{n} \subseteq 2^{J_{n}}$. By Lorentz's theorem, there exist sets $H_{n} \subseteq 2^{J_{n}}$, so that

$$
H_{n}+B_{n}=2^{J_{n}}
$$

$$
\left|H_{n}\right| \leq \frac{\ln \left(\left|B_{n}\right|\right)+1}{\left|B_{n}\right|} \cdot 2^{\left|J_{n}\right|} \leq \frac{1}{2^{n}} \cdot 2^{\left|J_{n}\right|}
$$

Define

$$
H=\left\{x \in 2^{\omega \times \omega}: \exists_{n}^{\infty} x\left\lceil J_{n} \in H_{n}\right\} .\right.
$$

It is easy to see that $H \in \mathcal{N}\left(2^{\omega \times \omega}\right)$. Let $t \in 2^{\omega \times \omega}$ be arbitrary. For each $n \in \omega$, choose $k(n) \in\left[0, K_{n}\right)$ such that $b_{n}^{(k(n))} \in H_{n}+\left(t \uparrow J_{n}\right)$. Notice that the relation 
$b_{n}^{(k)}$ can be identified with the partition $\left\{D_{n}^{(k)}, \tilde{D}_{n}^{(k)}\right\}$ of the set $I_{n}$, where $D_{n}^{(k)}=$ $\left\{a_{n}^{(l)}: l \leq k\right\}$ and $\tilde{D}_{n}^{(k)}=\left\{a_{n}^{(l)}: l>k\right\}$. Let us fix another partition $\left\{B_{n}^{(k)}, \tilde{B}_{n}^{(k)}\right\}$ of the set $\bigcup_{0 \leq k \leq K_{n}} A_{n}^{(k)}$, where $B_{n}^{(k)}=\bigcup_{l \leq k} A_{n}^{(l)}$ and $\tilde{B}_{n}^{(k)}=\bigcup_{l>k} A_{n}^{(l)}$. Finally, for $k=k(n)$, we define a partition $B$ by

$$
B=\{x \in A: x \cap\|s\| \neq \emptyset\} \cup\left\{\bigcup_{n \in \omega} B_{n}^{(k)}\right\} \cup\left\{\tilde{B}_{n}^{(k)}: n \in \omega\right\} .
$$

Clearly, $B \in(\omega)^{\omega}$ and $s \prec B \leq A$; thus, $B \in(s, A)$. Let $C \in(s, B)$ be arbitrary. Identify a partition $C$ with a function $\tilde{C}: \omega \times \omega \rightarrow 2$. Then for each $n \in \omega$, the relation $\tilde{C}\left\lceil J_{n}\right.$ is equal to $b_{n}^{(k(n))}$ or to $\mathbf{1}\left\lceil J_{n}\right.$, where $\mathbf{1}\left(\left\langle m_{1}, m_{2}\right\rangle\right)=1$. If we had $\forall_{n \in \omega}^{\infty} C\left\lceil J_{n}=\mathbf{1}\left\lceil J_{n}\right.\right.$, then $C$ would be finite which is impossible, since $C \in(\omega)^{\omega}$. Thus, we have $\exists_{n \in \omega}^{\infty} C \nmid J_{n}=b_{n}^{(k(n))}$. Hence, $C \in H+t$ which finishes the proof.

As a consequence we obtain the main theorem of this section.

Theorem 1.3. Suppose that $X$ is a strongly meager set in $2^{\omega \times \omega}$. Then it is dual Ramsey null.

Proof. Immediately follows from Lemma 1.2.

We will say that $X \subseteq 2^{\omega \times \omega}$ is a very meager $(V F C)$ set iff for each measure zero set $N$, there is a sequence $\left\{X_{n}\right\}_{n \in \omega}$ such that $X \subseteq \bigcup_{n \in \omega} X_{n}$ and $X_{n}+N \neq 2^{\omega \times \omega}$, for every $n \in \omega$.

Let us notice that the above argument gives us the following lemma.

Lemma 1.4. For any dual Ellentuck neighborhood $(s, A)$, there exists a measure zero set $H \in \mathcal{N}\left(2^{\omega \times \omega}\right)$ such that for each sequence $\left\{t_{n}\right\}_{n \in \omega} \subseteq 2^{\omega \times \omega}$, one can find $B \in(s, A)$ with $\forall_{n \in \omega}(s, B) \subseteq H+t_{n}$.

Corollary 1.5. Assume that $X$ is a very meager set in $2^{\omega \times \omega}$. Then it is dual Ramsey null.

Proof. Straightforward application of Lemma 1.4.

\section{Perfectly meager sets}

Throughout this part we write $f \prec g$, where $f, g \in \omega^{\uparrow \omega}$, iff $\forall_{n}^{\infty} f(n) \leq g(n)$. Depending on the context, a function $f \in \omega^{\uparrow \omega}$ is identified with the characteristic function of its range, and $t \in 2^{\omega}$ is often conflated with a function that increasingly enumerates the set $\{n: t(n)=1\}$. We assume that the reader is familiar with the notions of perfectly meager and universally meager sets (see, for example, G1], G2], and [NW1]). We recall below a definition of perfectly meager sets in the transitive sense which originally appeared in [NSW].

Definition 2.1. Let $X$ be a subset of the Cantor set $2^{\omega}$. We say that $X$ is an $A F C^{\prime}$ set (perfectly meager in the transitive sense) iff for every perfect set $P \subseteq 2^{\omega}$, one can find $F$, an $F_{\sigma}$ set containing $X$, such that for every $t \in 2^{\omega},(F+t) \cap P$ is meager in the relative topology of $P$.

We will use the following lemma which characterizes perfectly meager in the transitive sense subsets of $2^{\omega}$. 
Lemma 2.2. Let $X \subseteq 2^{\omega}$. The following conditions are equivalent:

(1) $X \notin A F C^{\prime}$.

(2) There exists a sequence $\left\{Q_{n}\right\}_{n<\omega}$ of perfect subsets of $2^{\omega}$ such that if $X \subseteq$ $\bigcup_{n<\omega} F_{n}, F_{n}=\overline{F_{n}}$, then there exist $n, m<\omega, t \in 2^{\omega}$ such that $Q_{m}+t \subseteq$ $F_{n}$.

Proof. See [NW1, Lemma 6.

Theorem 2.3. Let $X \subseteq 2^{\omega}$ be a set which satisfies the condition

$$
\forall_{f \in \omega^{\dagger \omega}}\left\{g \in \omega^{\uparrow \omega}: f \nprec g\right\} \cap X \in A F C^{\prime} .
$$

Then $X$ is an $A F C^{\prime}$ set.

Proof. The following lemma will be needed to prove our theorem.

Lemma 2.4. For every perfect set $Q \subseteq 2^{\omega}$, there exists $f_{Q} \in \omega^{\uparrow \omega}$ such that

$$
\forall_{t \in 2^{\omega}}(Q+t) \cap\left\{g \in \omega^{\uparrow \omega}: g \prec f_{Q}\right\} \neq \emptyset .
$$

Proof. Let $T \subseteq 2^{<\omega}$ be a perfect tree of the perfect set $Q$, i.e., $[T]=\left\{x \in 2^{\omega}\right.$ : $\forall_{k \in \omega} x\lceil k \in T\}=Q$. Choose a sequence $\left\{t_{s}: s \in 2^{<\omega}\right\} \subseteq T$ such that

$$
\forall_{s \in 2<\omega} \forall_{i \in 2} t_{s \frown\langle i\rangle} \supseteq t_{s} \frown\langle i\rangle \text {. }
$$

Define

$$
f_{Q}(l)=\left(\max _{s \in 2^{l}}\left|t_{s}\right|\right) .
$$

It is easy to see that $f_{Q} \in \omega^{\uparrow \omega}$.

Suppose that $t \in 2^{\omega}$. We construct a sequence $\left(s_{k}\right)_{k \in \omega}$ by induction as follows. Let $s_{0}=\emptyset$. Define $n_{k}=\left|t_{s_{k}}\right|$ and put $s_{k+1}=s_{k} \frown\left\langle 1-t\left(n_{k}\right)\right\rangle$. Finally, let $z=$

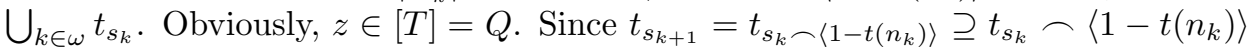
and $\left|t_{s_{k}}\right|=n_{k}$, we obtain that $t_{s_{k+1}}\left(n_{k}\right)=1-t\left(n_{k}\right)$; thus, $z\left(n_{k}\right)+t\left(n_{k}\right)=1$. Moreover, $\left|s_{k}\right|=k$ and therefore $n_{k}=\left|t_{s_{k}}\right| \leq \max _{s \in 2^{k}}\left|t_{s}\right|=f_{Q}(k)$. This implies that $z+t \prec f_{Q}$.

Fix $\left\{Q_{n}\right\}_{n \in \omega}$ to be a sequence of perfect sets, and choose $\bar{f} \in \omega^{\uparrow \omega}$ such that

$$
\forall_{n<\omega} \bar{f} \succ f_{Q_{n}} .
$$

Define

$$
f^{*}(n)=\bar{f}(n)+1 \text {. }
$$

Notice that the set $\left\{f \in[\omega]^{\omega}: f \succ f^{*}\right\}$ is an $F_{\sigma}$ set in the space $\omega^{\uparrow \omega}$. Let $\hat{F}$ be defined as

$$
\hat{F}=\left\{f \in[\omega]^{\omega}: f \succ f^{*}\right\} \cup[\omega]^{<\omega} .
$$

We easily see that $\hat{F}$ is an $F_{\sigma}$ set. Now let $t \in 2^{\omega}$ and $n \in \omega$ be given. There exists $q \in Q_{n}$ such that

$$
q+t \in\left\{g \in \omega^{\uparrow \omega}: g \prec f_{Q_{n}}\right\} \subseteq\left\{g \in \omega^{\uparrow \omega}: g \prec \bar{f}\right\} .
$$

We have that $q+t \notin \hat{F}$, since otherwise $q+t \in[\omega]^{\omega}$ and $q+t \succ f^{*}$. In this case $f^{*} \prec \bar{f}$ which contradicts the choice of $f^{*}$. This means that

$$
\forall_{n<\omega} \forall_{t \in 2} \omega Q_{n}+t \nsubseteq \hat{F} \text {. }
$$


By our assumption, $\left\{f \in \omega^{\uparrow \omega}: \neg\left(f^{*} \prec f\right)\right\} \cap X$ is an $A F C^{\prime}$ set, so by the characterization of $A F C^{\prime}$ sets, we obtain that there exists a sequence of closed sets $\left(F_{n}\right)_{n \in \omega} \subseteq 2^{\omega}$ such that

(1)

$$
\left\{f \in \omega^{\uparrow \omega}: \neg\left(f^{*} \prec f\right)\right\} \cap X \subseteq \bigcup_{n \in \omega} F_{n}
$$

$$
\forall_{n, m<\omega} \forall_{t \in 2 \omega} t+Q_{n} \not \subset F_{m} .
$$

We have that

$$
X \subseteq \hat{F} \cup\left[\left\{f \in \omega^{\uparrow \omega}: \neg\left(f \succ f^{*}\right)\right\} \cap X\right] \subseteq\left(\hat{F} \cup \bigcup_{n \in \omega} F_{n}\right) .
$$

Since

$$
\forall_{t \in 2^{\omega}} \forall_{m, n<\omega} Q_{n}+t \nsubseteq F_{m}
$$

and

$$
\forall_{t \in 2^{\omega}} \forall_{n<\omega} Q_{n}+t \not \subset \hat{F}
$$

it follows that $X$ is an $A F C^{\prime}$ set.

Conclusion 2.5. Assume $b=d$. Let $\left\{f_{\alpha}: \alpha<b\right\} \subseteq \omega^{\uparrow \omega}$ be a dominating family of elements from $\omega^{\uparrow \omega}$ such that

$$
\forall_{\alpha<\beta} f_{\alpha} \prec f_{\beta} .
$$

Then $\left\{f_{\alpha}: \alpha<b\right\}$ is an $A F C^{\prime}$ set.

Proof. This follows immediately from the fact that $\operatorname{non}\left(A F C^{\prime}\right) \geq \mathbf{d}$ (see $\left.[\mathbb{N}]\right)$.

For a finite set $s \in[\omega]^{<\omega}$ and an infinite $A \subseteq \omega$ with $\max (s)<\min (A)$, let $[s, A]=\left\{B \in[\omega]^{\omega}: s \subseteq B \subseteq s \cup A\right\}$. A tree $T \subseteq \omega^{<\uparrow \omega}$ is said to be a Laver tree iff for each $s$, with $\operatorname{stem}(T) \subseteq s$, the set of all immediate successors of $s$ is infinite (see [NW2] for details).

Definition 2.6. W shall say that $X \subseteq 2^{\omega}$ is a completely Ramsey null $\left(C R_{0}\right)$ set iff for every $[s, A]$, there is an infinite $B \subseteq A$ such that $[s, B] \cap X=\emptyset$. An $X \subseteq 2^{\omega}$ will be called an $l_{0}$-set iff for every Laver tree $T$, there exists a Laver tree $S \subseteq T$ with $\{\operatorname{ran}(x): x \in[S]\} \cap X=\emptyset$.

We have the following consequence of Theorem 2.3.

Theorem 2.7. Assume $C H$. Then there is an $A F C^{\prime}$ set $X \subseteq 2^{\omega}$ which is not a $C R_{0}$ set.

Proof. Assume that $\left\{g_{\alpha}\right\}_{\alpha<\omega_{1}}$ is an enumeration of $[\omega]^{\omega}$. Given $\left\{x_{\alpha}\right\}_{\alpha<\beta<\omega_{1}}$, so that

$$
\forall_{\alpha<\beta} x_{\alpha} \subseteq g_{\alpha}
$$

$$
\forall_{\alpha<\alpha^{\prime}<\beta} x_{\alpha} \prec x_{\alpha^{\prime}}
$$

find $x_{\beta} \subseteq g_{\beta}$ which satisfies

$$
\forall_{\alpha<\beta} x_{\alpha} \prec x_{\beta} .
$$

Clearly, $\left\{x_{\alpha}\right\}_{\alpha<\omega_{1}}$ is an $A F C^{\prime}$ set which is not $C R_{0}$. 
Corollary 2.8. Assume $C H$. Then there is an $A F C^{\prime}$ set $X \subseteq 2^{\omega}$ which is not an $l_{0}$-set.

Proof. First notice that for a given Laver tree $T$, one can find an $x \in[T]$ that dominates a countable family of elements from $\omega^{\uparrow \omega}$ and then repeat the above argument.

Motivated by Theorem 2.3 we introduce the following notion.

Definition 2.9. We say that a $\sigma$-ideal $\mathcal{I}$ of subsets of $2^{\omega}$ satisfies condition ( $\left.\ddagger\right)$ if and only if the following holds.

For every $X \subseteq 2^{\omega}$, if

$$
\forall f \in \omega^{\uparrow \omega}\left\{g \in \omega^{\uparrow \omega}: \neg(f \prec g)\right\} \cap X \in \mathcal{I},
$$

then $X \in \mathcal{I}$.

Let us denote for brevity the set $\left\{g \in \omega^{\uparrow \omega}: \neg(f \prec g)\right\}$ as $G_{f}$.

Observation 2.10. The $\sigma$-ideals $A F C$, of perfectly meager sets, and $\overline{A F C}=U M$, of universally meager sets, satisfy condition ( $\ddagger)$.

Proof. Suppose that a set $X \subseteq 2^{\omega}$ satisfies condition (1) from above for $\mathcal{I}=A F C$. Let $Q$ be a perfect set and a sequence $\left(Q_{n}\right)_{n \in \omega}$ be an enumeration of all nonempty elements of the class $\left\{Q \cap[s]: s \in 2^{<\omega}\right\}$. For each $n \in \omega$, choose a function $f_{n} \in \omega^{\uparrow \omega} \cap Q_{n}$. Let $f^{*} \in \omega^{\uparrow \omega}$ be such that $\forall_{n \in \omega} f_{n} \prec f^{*}$ and define $\bar{f}(n)=f^{*}(n)+1$. Assume that there is an $n \in \omega$ such that $Q_{n} \subseteq Q \backslash G_{\bar{f}}$. Then $Q_{n} \subseteq Q \backslash G_{\bar{f}} \subseteq$ $Q \backslash G_{f_{n}+1}$, since $f_{n}+1 \prec \bar{f}$. But $f_{n} \in Q_{n}$, so $f_{n} \notin G_{f_{n}+1}$ and hence $f_{n}+1 \prec f_{n}$ which is a contradiction. This finishes the proof that $Q \backslash G_{\bar{f}} \in \mathcal{M G \mathcal { R }}(Q)$, the set of meager subsets of $Q$ in the relative topology of $Q$. Therefore, $X \in A F C$.

We now proceed to prove that $\overline{A F C}$ satisfies ( $\ddagger)$. To begin this proof we quote a characterization of $\overline{A F C}$ sets which may be found in $B$.

Characterization 2.11 (T. Bartoszyński $[\mathrm{B}]$ ). A set $X$ is an $\overline{A F C}$ set iff for every sequence of perfect sets $\left(P_{n}\right)_{n \in \omega}$ there exists an $F_{\sigma}$ set $E$ such that $X \subseteq E$ and $E$ is meager in $P_{n}$, for every $n \in \omega$.

Let $\left(P_{n}\right)_{n \in \omega}$ be a sequence of perfect sets. For $n \in \omega$, let $\left(Q_{n, m}\right)_{m \in \omega}$ be an enumeration of all nonempty elements of the family $\left\{P_{n} \cap[s]: s \in 2^{<\omega}\right\}$. For every $n, m \in \omega$ choose $f_{n, m} \in \omega^{\uparrow \omega} \cap Q_{n, m}$. Let $f^{*} \in \omega^{\uparrow \omega}$ be such that $\forall_{n, m \in \omega} f_{n, m} \prec f^{*}$ and define $\bar{f}=f^{*}+1$. Suppose that $Q_{n, m} \subseteq 2^{\omega} \backslash G_{\bar{f}}$. Since $f_{n, m} \notin G_{\bar{f}}, f^{*}+1 \prec f_{n, m}$ and hence $f^{*}+1 \prec f_{n, m} \prec f^{*}$, which is impossible. Therefore, $\forall_{n \in \omega}\left(2^{\omega} \backslash G_{\bar{f}}\right) \cap P_{n} \in$ $\mathcal{M G R}\left(P_{n}\right)$. Let us denote $E=2^{\omega} \backslash G_{\bar{f}}$. By assumption, we have $G_{\bar{f}} \cap X \in \overline{A F C}$, so there is an $F_{\sigma}$ set $\tilde{E}$ such that $G_{\bar{f}} \cap X \subseteq \tilde{E}$ and $\forall_{n \in \omega} P_{n} \cap \tilde{E} \in \mathcal{M G R}\left(P_{n}\right)$. Define $E^{*}=E \cup \tilde{E}$. Obviously, $E^{*}$ is an $F_{\sigma}$ set. Moreover, $X \subseteq\left(X \cap G_{\bar{f}}\right) \cup\left(2^{\omega} \backslash G_{\bar{f}}\right) \subseteq$ $\tilde{E} \cup E=E^{*}$ and for each $n \in \omega, E^{*} \cap P_{n}=\left(\tilde{E} \cap P_{n}\right) \cup\left(E \cap P_{n}\right) \in \mathcal{M G R}\left(P_{n}\right)$. This proves that $X \in \overline{A F C}$.

We finish this paper with the following question we are unable to answer.

Problem 2.12. Is it true that the $\sigma$-ideal of $\lambda^{\prime}$ subsets of $2^{\omega}$ satisfies condition $(\ddagger)$ ? 


\section{ADDED IN PROOF}

A. Miller proved recently (see $[\mathrm{M}]$ ) that it is independent from the ZFC axioms whether or not every $\lambda^{\prime}$-set satisfies condition $(\ddagger)$.

\section{REFERENCES}

[B] T. Bartoszyński Remarks on small sets of reals, preprint

[BJ] T. Bartoszyński, H. Judah, Borel images of sets of reals, Real Analysis Exchange 20(2) (1994/5), 536 - 558. MR 96k:04005

[CS] T.J. Carlson, S.G. Simpson, A dual form of Ramsey's Theorem, Advances in Mathematics 53 (1984), 265 - 290. MR 85h:04002

[G1] E. Grzegorek, Always of the first category sets, Proceedings of the 12th Winter School on Abstract Analysis Srni(Bohemian Weald), 15-29 January, 1984, Section of Topology, Supplemento ai Rend. Circ. Mat. Palermo, Serie II-numero 6-1984, 139-147. MR 86a:00004

[G2] E. Grzegorek, Always of the first category sets (II), Proceedings of the 13th Winter School on Abstract Analysis Srni(Bohemian Weald), 20-27 January, 1985, Section of Topology, Supplemento ai Rend. Circ. Mat. Palermo, Serie II-numero 10-1985, 43-48. MR 88j:54054

[L] G.G. Lorentz, On a problem of additive number theory, Proceedings of the American Mathematical Society 5 (1954), 838 - 841. MR 16:113f

[M] A. W. Miller, On $\lambda^{\prime}$-sets, preprint, 2003.

[N] A. Nowik, Remarks about transitive version of perfectly meager sets, Real Analysis Exchange 22(1) (1996/97) 406 - 412. MR 97m:54043

[NSW] A. Nowik, M. Scheepers, T. Weiss, The algebraic sum of sets of real numbers with strong measure zero sets. Journal of Symbolic Logic 63 (1998), 301 - 324. MR 99c:54049

[NW1] A. Nowik, T. Weiss, Not every Q-set is perfectly meager in the transitive sense, Proceedings of The American Mathematical Society 128(496), No 10 (October 2000), 3017 - 3024. MR 2000m:03116

[NW2] A. Nowik, T. Weiss, Strongly meager sets of real numbers and tree forcing notions. Proceedings of The American Mathematical Society 110, No 4 (2002), 1183-1187. MR 2002j:03049

Institute of Mathematics, University of Gdańsk, Wita Stwosza 57, 80 - 952 Gdańsk, POLAND

E-mail address: matan@julia.univ.gda.pl

Current address: Institute of Mathematics, Polish Academy of Sciences, Abrahama 18, 81-825 Sopot, Poland

E-mail address: nowik@impan.gda.pl

Institute of Mathematics, WSRP, 08-110 Siedlce, Poland

E-mail address: weiss@wsrp.siedlce.pl 